Infinite Dimensional Analysis, Quantum Probability

and Related Topics

Vol. 22, No. 4 (2019) 1999001 (2 pages)

(C) World Scientific Publishing Company

DOI: $10.1142 / \mathrm{S} 0219025719990017$

\title{
Author index (Vol. 22)
}

Accardi, L. \& Lu, Y.G., The $q q$-bit (III): Symmetric $q$-JordanWigner embeddings

Accardi, L., Ji, U. C. \& Saitô, K., Characterization of $S$-transform for general construction of infinite-dimensional distributions

Accardi, L., see Rebei

Albeverio, S. \& Steblovskaya, V., Asymptotics of Gaussian integrals in infinite dimensions

Allahverdiev, B. P. \& Tuna, H., TitchmarshWeyl theory for $q$-Dirac systems

Bolaños-Servin, J. R., Carbone, R. \& Quezada, R., Structure and block representation for circulant quantum processes

Bouali, M., Olshanski spherical pairs of semigroups type

Carbone, R., see BolañosServin

Cheng, L.-J. \& Zhang, S.-Q., Weak Poincaré inequalities for convolution probabilities measures

Chesneau, C., see Jamal

Dehghan, H., see Rezapour

Dolai, D. R., see Mallick

Fagnola, F. \& Quezada, R., A characterization of quantum Markov semigroups of weak coupling limit type
22 (2019) 1850023

22 (2019) 1950015

22 (2019) 1950025

22 (2019) 1950004

22 (2019) 1950010

22 (2019) 1950017

22 (2019) 1950021

22 (2019) 1950017

22 (2019) 1950019

22 (2019) 1950027

22 (2019) 1950003

22 (2019) 1950012

22 (2019) 1950008
Ferrari, S., Sobolev spaces with respect to a weighted Gaussian measure in infinite dimensions

Gao, M., Compound bifree Poisson distributions

Hao, Zh., see Popa

Hayase, T., Identifiability of parametric random matrix models

Hiai, F. \& Lim, Y., Convergence theorems for barycentric maps

Jamal, F. \& Chesneau, C., A new family of polynoexpo-trigonometric distributions with applications

James, M. R., see Vladimirov

Ji, U. C., see Accardi

Lenczewski, R., Conditionally monotone independence and the associated products of graphs

Li, S., Liu, W. \& Xie, Y., Ergodicity of 3D Leray$\alpha$ model with fractional dissipation and degenerate stochastic forcing

Lim, Y., see Hiai

Liu, W. \& Zhong, P., Free-Boolean independence with amalgamation

Liu, W., see $\mathrm{Li}$

Lu, Y.-G., see Accardi

Makasu, C., A stochastic Gronwall lemma revisited

Mallick, A. \& Dolai, D. R., Spectral statistics
22 (2019) 1950023

22 (2019) 1950026

22 (2019) 1950014 22 (2019) 1950024

22 (2019) 1950018

22 (2019) 1950016

22 (2019) 1950027

22 (2019) 1950020

22 (2019) 1950015

22 (2019) 1950002

22 (2019) 1950028 22 (2019) 1950002 22 (2019) 1850023

22 (2019) 1950007 22 (2019) 1950016 
for one-dimensional Anderson model with unbounded but decaying potential

Mohan, M. T. \& Sritharan, S. S., Stochastic Navier-Stokes equations perturbed by Lévy noise with hereditary viscosity

Osgooei, E., see Rezapour

Pandiscia, C., Factorization of an adjontable Markov operator

Petersen, I. R., see Vladimirov

Popa, G. \& Stan, A. I., A characterization of probability measures in terms of semi-quantum operators

Popa, M. \& Hao, Zh., An asymptotic property of large matrices with identically distributed Boolean independent entries

Quezada, R., see BolañosServin

Quezada, R., see Fagnola Rahimi, A., see Rezapour Rebei, H., Accardi, L. \& Taouil, H., Tensor Bogolyubov representations of the renormalized square of white noise (RSWN) algebra

Rezapour, R., Rahimi, A., Osgooei, E. \& Dehghan, H., Controlled weaving frames in Hilbert spaces

Saitô, K., see Accardi

Slaoui, M. \& Tudor, C.

22 (2019) 1950012

22 (2019) 1950006

22 (2019) 1950003

22 (2019) 1950013

22 (2019) 1950020

22 (2019) 1950009

22 (2019) 1950024

22 (2019) 1950017

22 (2019) 1950008

22 (2019) 1950003

22 (2019) 1950025

22 (2019) 1950003
A., The linear stochastic heat equation with Hermite noise

Sritharan, S. S., see Mohan

Stan, A. I., see Popa

Steblovskaya, V., see Albeverio

Taouil, H., see Rebei

Tate, T., Eigenvalues, absolute continuity and localizations for periodic unitary transition operators

Teretenkov, A. E., Irreversible quantum evolution with quadratic generator: Review

Tudor, C. A., see Slaoui

Tuna, H., see Allahverdiev

Vladimirov, I. G., Petersen, I. R. \& James, M. R., Parametric randomization, complex symplectic factorizations, and quadraticexponential functionals for Gaussian quantum states

Volkov, B. O., Lévy differential operators and Gauge invariant equations for Dirac and Higgs fields

Wu, W., An approximation property for operator systems

Xie, Y., see Li

Zhang, S.-Q., see Cheng

Zhong, P., see Liu
22 (2019) 1950015

22 (2019) 1950022

22 (2019) 1950006

22 (2019) 1950009

22 (2019) 1950004

22 (2019) 1950025

22 (2019) 1950011

22 (2019) 1930001

22 (2019) 1950022

22 (2019) 1950010

22 (2019) 1950020

22 (2019) 1950001

22 (2019) 1950005

22 (2019) 1950002

22 (2019) 1950019

22 (2019) 1950028 